\title{
The role of neuroimaging in translational cognitive neuroscience
}

\author{
Roland Zahn, MD PhD \\ a The University of Manchester, School of Psychological Sciences, Neuroscience and \\ Aphasia Research Unit, Manchester, M13 9PL, UK \\ Correspondence to: \\ Dr. Roland Zahn (see address above) \\ Tel: +44-(0)161-27-57338 \\ Fax: +44-(0)161-27-52873 \\ e-mail: roland.zahn@manchester.ac.uk
}

This article will appear in the Special issue (2009):

Topics in MRI (copyright reserved by the publishers):

"MRI of Cognitive Disorders", ed. by Jorge Moll 


\begin{abstract}
Despite the current enthusiasm for neuroimaging as a key method in translational neuroscience, there is a lack of debate about the nosological framework within which neuroimaging measures should be related to diagnostic categories. Here, the aim is to stimulate a debate about the role of cognitive neuroscience and neuroimaging in mediating between molecular/genetic, clinical diagnostic and symptom-based descriptions of neuropsychiatric disorders. The diagnostic role of neuroimaging in translational neuroscience is stressed, namely to be combined with cognitive measures in order to define cognitiveanatomical syndromes as an intermediate diagnostic category which mediates between clinical diagnoses and psycho-reactive as well as neurobiological aetiological factors. This multilevel approach will be illustrated by reviewing recent insights into the cognitiveanatomical basis of inappropriate social behaviour and social knowledge in frontotemporal dementia and by discussing its implications for the study of neuropsychiatric disorders such as major depressive disorder in which neuroanatomical abnormalities are more subtle.
\end{abstract}

Key words: Translational Medicine; Nosology; Psychiatry; Neurology; Major Depression; Frontotemporal Dementia; Social Cognition; Social Knowledge; Moral Emotions; Moral Sentiments; Guilt 


\section{Introduction}

Translational neuroscience is part of the recently launched field of translational medicine, defined as the effort to use basic research advances (i.e. studies of biological processes) to develop new therapies or medical procedures ${ }^{1}$. Here, I argue that the success of translational neuroscience will depend on linking basic insights from cognitive neuroscience which often relies on neuroimaging in healthy populations with clinical neuropsychiatric disorders. The methodological challenges of translating "basic" cognitive neuroscience into clinical neuropsychiatry are significant and therefore I propose a methodological framework labelled as "translational cognitive neuroscience" to highlight its bridging function between "translational molecular neuroscience" and clinical neuropsychiatry. For a definition of the aims of basic cognitive neuroscience, translational cognitive neuroscience and clinical neuropsychiatry see Figure 1.

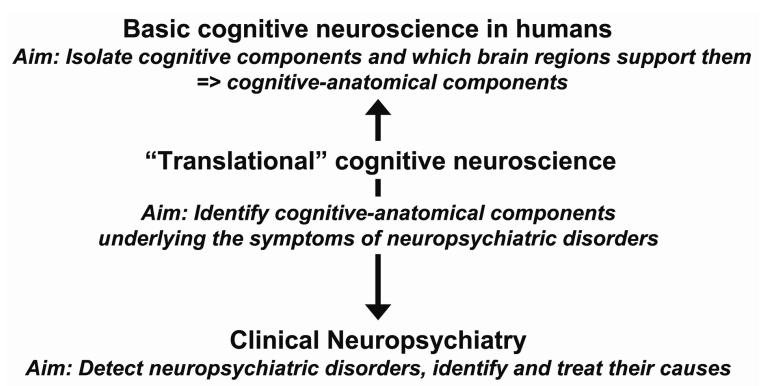

Fig 1. The aims of basic, translational cognitive neuroscience and clinical neuropsychiatry are depicted.

The translational cognitive neuroscience approach proposed here aims to identify the cognitive-anatomical components which are disrupted in neuropsychiatric disorders. Cognitive-anatomical components refer to cognitive (including emotional) components linked with particular neuroanatomical substrates. The term "neuropsychiatric" disorders is used here to refer to all central nervous system disorders irrespective of their cause, whether due to marked macro-anatomical abnormalities or other neurobiological factors and irrespective of the degree to which they require additional reaction to psychological (including culturally-shaped) experiences and are thus modulated by learning. The proposed focus on the identification of cognitive-anatomical underpinnings of neuropsychiatric disorders is a subtle, but important difference from translational neuroscience in its currently practised form which uses cognitive neuroscience methods such as neuropsychological test 
examination or functional MRI mainly to replace or refine clinical diagnoses when making correlations with molecular or genetic factors of aetiology.

As Kendler pointed out ${ }^{2}$, there is still a divide within psychiatric nosology (i.e. the science of defining and classifying psychiatric illnesses) between "hard reductionism ('all psychiatric illness is best explained solely in terms of molecular neuroscience') and hard emergentism ('all psychiatric illness is best explained solely in terms of specified mental or social mechanisms and cannot be deduced from biology')". Kendler's suggestion is to find a middle ground between these extremes by using a multilevel approach which aims to decompose first the simple subsystems at different levels (e.g. molecular, psychological) and then to study their complex interactions, but without aiming to find one-to-one correspondences between units at different levels ${ }^{2}$. Here, I suggest a methodological framework for implementing such a multilevel approach to understand the relationship of neurobiological and psycho-reactive pathogenetic factors with symptoms of neuropsychiatric disorders and highlight the critical role of neuroimaging.

Translational cognitive neuroscience is related to the "cognitive neuropsychiatry" approach proposed by Halligan \& David3, defined as aiming to establish "the functional organization of psychiatric disorders within a framework of cognitive neuropsychology and linking this framework to relevant brain structures and their pathology." Cognitive neuropsychology is a branch of cognitive neuroscience emphasizing the importance of single case analyses and establishing the separation of cognitive components by demonstrating their dissociation in patients with neurological disorders 4 . The cognitive neuropsychological method has led to a wealth of valuable data and to refined models of higher cognitive functions. The classical models of cognitive neuropsychology, however, did not primarily aim to identify the anatomical localization of cognitive components which was partly due to the difficulty of establishing simple lesion localization-cognitive function associations in case series, especially when relying on low spatial resolution imaging methods such as computed tomography (CT) and Single-Photon-Emission-Computed-Tomography (SPECT) in the early days of cognitive neuropsychology.

Modern structural MRI, Positron Emission Tomography (PET), MR-spectroscopy, MRdiffusion weighted imaging, perfusion MRI together with larger numbers of cases have improved our ability to link specific cognitive functions with localization of lesions. Perfusion MRI in acute stroke patients, for example, has been recently used to associate specific cognitive components of language abilities with hypoperfusion in particular brain regions 5 . 
With the advent of functional MRI in the 1990ies, a novel way of establishing associations between anatomical brain regions and their cognitive functions became available. This has contributed to the emergence of "cognitive neuroscience" as a field which integrates insights from the basic neurosciences in animals with the study of higher cognition in humans using either neuroimaging (including neurophysiological brain mapping such as electroencephalography (EEG)) in healthy people or neuropsychological test examination in patients with neuropsychiatric disorders ${ }^{6}$.

Although neuroimaging and neuropsychological measures are widely used within the context of translational neuroscience, the arising theoretical and methodological questions of how to meaningfully do so are elusive. Here, the following core questions will be discussed:

1. What are the best clinical measures for translational neuroscience research: Clinical diagnoses as defined by international classification systems, clinical symptoms, syndromes, or surrogate imaging biomarkers?

2. What are the necessary levels of modelling the pathogenesis of neuropsychiatric disorders: Psycho-social, cognitive, cognitive-anatomical, or molecular?

3. How many levels of description should successful models of neuropsychiatric disorders have?

4. How should different levels of description be related with each other in translational neuroscience research?

5. What is the role of neuroimaging for aetio-pathogenetic models of neuropsychiatric disorders?

In order to address these questions, this paper starts with briefly describing current approaches and controversies regarding clinical measures and surrogates in translational research, and will then summarize different approaches to aetio-pathogenetic models of neuropsychiatric disorders in general. The translational cognitive neuroscience approach will be illustrated by applying it to the understanding of the pathogenesis of inappropriate social behaviour in frontotemporal dementia (FTD) syndromes. Further, I will propose how these methodological principles could be applied to understand neuropsychiatric disorders such as major depression that do not exhibit the same degree of macro-anatomical abnormalities as can be found in dementias or cognitive disorders due to injury, inflammation, stroke or brain tumours. The final section summarizes the main arguments and gives future perspectives for translational cognitive neuroscience research. 


\section{Clinical measures in translational neuroscience research}

One of the keys to successful translational neuroscience are appropriate measures of the clinical disorder which is the object of aetio-pathogenetic understanding or the target of therapeutic intervention. Neuropsychiatric symptoms are abnormal experiences or behaviours which cause significant suffering of either the patient or people whom the patient interacts with. It is this latter component of symptoms that makes a consideration of the socio-cultural and personal norms that underlie definitions of abnormality and suffering inevitable in any nosology of neuropsychiatric disorders. Transcultural studies are therefore indispensible in order to establish transculturally stable associations between abnormalities of experiences or behaviour and suffering. But even for disorders such as Alzheimer's dementia or major depressive disorder that have been established to be associated with a set of core symptoms across cultures, there remains the uncertainty of different diagnostic thresholds depending on cultural and personal variation in subjective experience, communication and assessments of the symptom as well as different cultural and personal perceptions of thresholds of tolerable suffering. For example, the diagnosis of dementia according to ICD-10 (International Classification of Disorders, $10^{\text {th }}$ edition7) criteria requires a significant reduction in daily life functioning. This threshold is known to be influenced by the demands on cognitive functioning for the person with dementia in daily life and cultural differences will largely influence when patients and carers think of the problem as significant.

One suggested solution to this threshold problem has been to abolish diagnostic thresholds and think of symptom dimensions in order to increase sensitivity of correlations between neurobiological markers and clinical measures ${ }^{8}$. One danger of abolishing diagnostic thresholds for symptoms and their combinations into clinical syndromes is that they may loose diagnostic specificity. For example, if clinicians were to call any type of low mood, "depressive mood", irrespective of whether it causes significant suffering or is present persistently most of the day and more days than not, we would inevitably loose some of the core defining features of depressive or dysthymic mood as opposed to low mood occurring in everyday life and not associated with high degrees of psychosocial impairment. Subthreshold neuropsychiatric symptoms irrespective of their pathological value (i.e. how much they cause suffering taking the literal meaning of the greek word "pathos") may be more sensitive indicators of disorders and may be more transculturally stable, but may lack specificity and 
therefore be disadvantageous for translational cognitive neuroscience and clinical diagnostic purposes.

Measures of neuropsychiatric symptoms rely on self-rating scales or structured, semistructured or free interviews of clinicians with patients and carers. The best symptom descriptions are phenomenological in that they are as free as possible of prior assumptions and interpretations and try to depict the patient's subjective experience as accurately as possible ${ }^{9}$. Although phenomenological qualities of symptoms can be assessed with good interrater reliability ${ }^{10}$, the reliable use of semi-structured interviews which aim at clinical diagnoses rather than refined symptom description can probably be learned more easily (e.g. Structured Clinical Diagnostic Interview for American Psychiatric Association Diagnostic Statistical Manual (DSM)-IV ${ }^{11}$ ). Abnormal behaviour rather than experience can be measured with high inter-rater reliability using structured interviews with carers of patients (e.g. ${ }^{12}$ ). Despite the high inter-rater reliability of many neuropsychiatric diagnoses, the problem of the validity of many of our diagnoses remains as elusive as at the beginning of modern neuropsychiatry in the $19^{\text {th }}$ century.

The difficulty of validity is the difficulty of defining a gold standard against which to validate clinical diagnoses. Usually the way we validate clinical diagnoses is by testing whether they are specifically associated with a certain type of aetiology. Clinical diagnoses mostly consist of a combination of symptoms (i.e. a clinical syndrome) with some aetiological constraints. For example, the exclusion of mood changes due to substance abuse or organic brain damage in DSMIV is the aetiological constraint when making a diagnosis of major depressive disorder based on a combination of clinical symptoms. Neuropsychiatric disorders with gross macroanatomical abnormalities such as dementias seem to deal better with the problem of validity at first sight, because their aetiology seems to be more clearly defined. As our discussion below on current controversies of subclassification of dementias shows, however, the principle problem remains the same.

The problem of validity of clinical diagnoses has led to the widely held notion that translational neuroscience will benefit from advances in molecular neuroscience and genetics allowing us to replace clinical diagnoses with biomarkers of the neurobiological change. Kendler has pointed out, however, that the presence of a neurobiological variation in itself, even when heritable, does not point to its validity as a pathological entity ${ }^{13}$. Variations in height, hair colour and nose size, for example, are heritable ${ }^{13}$ and could co-occur in families, one could also define a statistical threshold of abnormality from a norm population, but this 
would not inform us about the pathological value of this syndrome. I will describe below how a multilevel approach, as has been suggested by Kendler ${ }^{2}$, could be implemented in order to relate symptoms with clinical diagnoses and neurobiological factors of aetiology.

\section{Multilevel vs. dual-level aetio-pathogenetic models of neuropsychiatric disorders}

A widely practiced approach to translational neuroscience is to focus primarily on two levels of description, one measuring the phenotype or expression of the illness and another measuring the molecular or genetic cause (aetio-pathogenetic factor).

Functional MRI measures have been suggested as “intermediate phenotypes" for correlations with individual variations on gene polymorphisms in psychiatric disorders ${ }^{14}$. The intermediate phenotype approach seeks to replace clinical syndromes with more neurobiologically valid measures such as functional MRI in order to attain closer correlations between genetic variations and their phenotypic expression in humans. Imaging measures such as fMRI or structural MRI have also been suggested as "surrogate markers" for clinical manifestations of neuropsychiatric disorders ${ }^{15}$ or for predicting response to drug treatment ${ }^{16}$, 17. The purpose of volumetric MRI surrogate markers in neurodegenerative diseases ${ }^{15}$, for example, is to give a more sensitive index of disease progression for clinical trials than clinical symptoms or neuropsychological tests could deliver.

Some of the intermediate phenotype and surrogate biomarker approaches may suggest a reductionist model for the use of neuroimaging in translational neuroscience (Fig 2a). The role of neuroimaging according to a reductionist view would be to deliver a quantitative measure that can replace the clinical diagnosis ${ }^{15}$. These neuroimaging biomarkers could be of great benefit to drug development ${ }^{16,17}$, however, up to now there is no available neuroimaging biomarker that has proven to be highly predictive of clinical outcome ${ }^{17}$. The clinical diagnosis/biomarker could be re-iteratively refined in order to correspond as unambiguously as possible to a molecular cause of the disorder. A recent example of successes with this reductionist approach was that different clinical dementia syndromes associated with different distributions of frontal and/or temporal focal abnormalities as identified on MRI had a relatively high predictive value for specific neuropathological changes on microscopy after death which could further be related to certain molecular pathogenetic pathways ${ }^{18}$. 
The reductionist model of translational neuroscience has the advantage of being easier to use and convey to clinicians. The disadvantage of re-defining clinical diagnostic categories by neuropathological or molecular correlations is that clinical classifications may become quite unstable and that diagnostic categories may become increasingly fragmented, the more different molecular pathogenetic causes we will be able to detect. Another more fundamental problem of reductionist approaches is that different molecular causes can lead to the same regional distribution of pathology disrupting the same cognitive-anatomical components and lead to identical clinical symptoms. For example, progressive behavioural changes due to ventral frontal atrophy can arise by Alzheimer's disease (AD)-typical microscopical changes (neurofibrillary plaques and tangles) with an atypical regional distribution of pathology in the frontal rather than the medial temporal lobe ${ }^{19}$. A reductionist approach to translational neuroscience would aim to define the diagnosis of frontotemporal dementia (FTD) in a way to exclude patients with AD-typical microscopical changes with the argument that the molecular pathogenesis resembles more typical Alzheimer's disease than other forms of FTD. This argument is well understandable when considering pharmacological treatments aimed at the molecular cause of a disease. However, regarding neuroimaging, neuropsychology, symptoms, management and prognosis, the patient with AD-typical microscopical changes affecting frontal and anterior temporal lobes may be more comparable with other FTD patients despite their differences in molecular pathology.

A multilevel approach to the nosology of neuropsychiatric disorders, as depicted in Figure 2b, allows for complex and ambiguous relationships between molecular causes, regional macroanatomical distribution of abnormalities and clinical symptoms. Instead of aiming to replace clinical diagnoses with neuroimaging or neuropsychological markers, this approach aims at separating: 1) clinical syndromes as a combination of clinical symptoms defined by a clinician's interview, and 2) clinical diagnoses as a combination of clinical syndromes with certain aetiological constraints, from 3) "cognitive-anatomical" syndromes at an intermediate level which allow correlation with regional distribution of neuropathology. The regional distribution of neuropathology is then related to molecular and genetic causes. Cognitiveanatomical syndromes are the result of translational cognitive neuroscience research and consist of a combination of intact, impaired, and potentially compensatorily enhanced cognitive-anatomical components. Neuroimaging and neuropsychological test examination in healthy people and in people with different types of disorders are used to identify dissociable cognitive-anatomical components. These are then used to build models which explain changes in subjective experiences and abilities in a personal and cultural context based on 
disruption of particular cognitive-anatomical components with a particular time frame and type of damage.

In order to illustrate the classical (reductionist) and the proposed translational cognitive neuroscience approach, take for example clinical syndromes characterized by slowly progressive cognitive impairments acquired in adulthood. When defining a clinical syndrome, one takes the time course of development of symptoms into account, the relevance to daily life functioning, but also the exact combination of symptoms and lead symptoms ${ }^{20}$. There are different lead symptoms which can be used to distinguish different clinical syndromes. For example, if a patient shows slowly progressive changes in social behaviour in a way thought of as inappropriate by their carers and departing from the patient's previous social conduct, but the patient is initially well able to remember daily life events, has no problems with spatial orientation, one will classify this patient as showing a slowly progressive behavioural syndrome. Many clinicians will directly come to a clinical diagnosis based on the clinical syndrome, but which entails a number of aetiological constraints. For example, one may diagnose a behavioural variant of FTD according to the Lund-Manchester consensus criteria ${ }^{21}$ (I will use the term "frontotemporal dementia or FTD" instead of the original "frontotemporal lobar degeneration" throughout the text as the superordinate label for the clinical diagnoses of behavioural variant FTD, semantic dementia and progressive nonfluent aphasia). But this requires exclusion of non-neurodegenerative aetiologies, such as brain tumours or inflammatory causes for example. In most clinical settings this is achieved by standard neuroimaging techniques such as a non-contrast computed tomography (CT) and blood tests. Recent evidence-based clinical guidelines state that visual inspection of regional distribution of abnormalities on Single-Photon-Emission-Computed-Tomography (SPECT)/ 18Fluorodeoxyglucose-Positron-Emission-Tomography (18-FDG-PET) or MRI are recommended to improve differential diagnosis ${ }^{22}$. Standard neuroimaging may show abnormalities in ventral frontal cortex. The role of standard neuroimaging is thus to help to consolidate the clinical diagnosis.

The role of neuroimaging in a surrogate biomarker approach ${ }^{15}$ here would be to replace the clinical diagnosis by, for example, a measure of atrophy in the ventral frontal lobe. In contrast, a multi-level translational cognitive neuroscience approach would use neuroimaging techniques and neuropsychological test examination to identify the intact and impaired cognitive-anatomical components in patients with a given clinical diagnosis such as the behavioural variant of FTD. The combination of intact and impaired cognitive components can then be used to define a cognitive-anatomical syndrome which explains the clinical 
syndrome on the basis of the regional distribution of pathology in the brain and the type of lesion. For example, a slowly progressive neurodegenerative lesion to the ventral frontal lobe will lead to different cognitive symptoms than a rapid onset inflammatory lesion ${ }^{23}$, even if the anatomical distribution of the lesion is similar. This is because of the difference in the ability of the remaining brain tissue to reorganize its functions in order to compensate for lesioned tissue. The main advantage of introducing a new intermediate level of cognitive-anatomical syndromes is that the same clinical diagnosis can be related with different cognitiveanatomical syndromes and the same cognitive-anatomical syndrome can be associated with different clinical diagnoses. This allows different aetiologically and symptomatically similar, but cognitive-anatomically different syndromes to be recognized.

For example, the clinical diagnosis of the behavioural variant of FTD is associated with variable degrees of ventral frontal and anterior temporal lobe lesions and, as I will further describe in the next section, the same lead symptom of the associated clinical syndrome, namely inappropriate social behaviour, can be associated with disruption of different cognitive-anatomical components.

Different cognitive-anatomical variants of the same clinical diagnosis could be related with post-mortem neuropathology and regional distribution of microscopically or histochemically detected changes. But there could be also identical histochemistry between different cognitive-anatomical variants.

The reductionist approach to translational neuroscience would be to redefine the clinical diagnosis in order to map more closely onto the histochemical changes. This redefinition of clinical diagnoses could lead to higher prediction rates of histochemical changes for the clinician, but it will inevitably fail to recognize cases in which the histochemical changes are equally distributed anatomically, but are of a different kind. For example, it has been shown that almost $50 \%$ of cases with progressive nonfluent language impairments show AD-typical histochemical and microscopical changes ${ }^{19}$. Furthermore, it has been demonstrated that the regional distribution of pathology is determined by the clinical syndrome rather than the type of histochemical changes ${ }^{24}$.

Clinical diagnoses are always partially aetiologically defined, but are often aetiologically heterogenous in order to allow grouping of similar clinical presentations. This mixture of symptom-based and aetiological definition of clinical diagnoses emphasizes classification of disorders according to prognosis, symptomatic management and treatment. For example, a 
patient with progressive nonfluent speech problems due to left perisylvian and insular cortical degeneration, but intact spatial orientation and memory will profit from the diagnosis of progressive nonfluent aphasia by knowing that this disorder has a different course than classical Alzheimer's dementia and that they will need different management and treatment. For example, swallowing problems with aspiration pneumonia are a common problem which needs attention by speech and language therapists. Depending on pragmatic/clinical considerations one could redefine the clinical diagnosis based on different cognitiveanatomical variants, but only if the different cognitive-anatomical variants are significantly different in management and prognosis.

In the next section, I will illustrate the use of a translational cognitive neuroscience approach to core symptoms of the behavioural variant of FTD. 


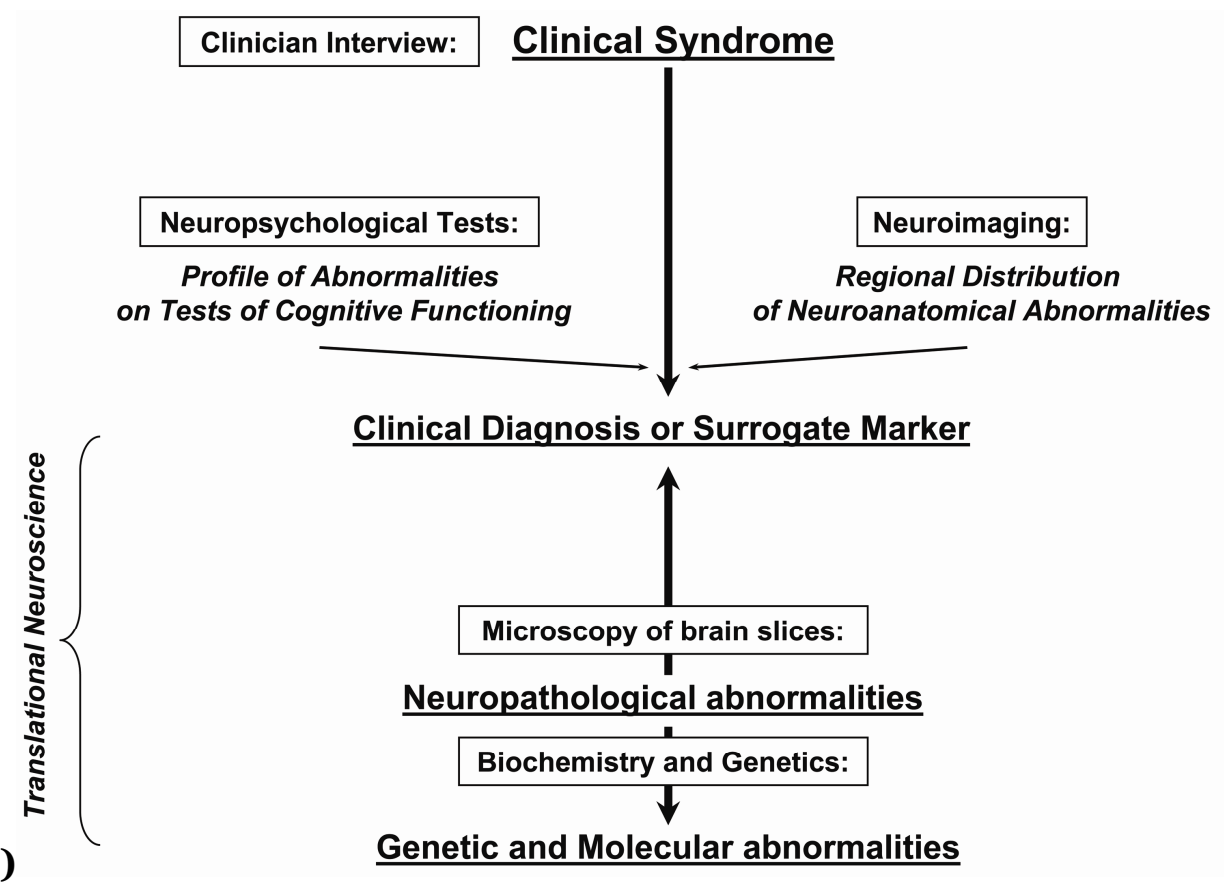

a)

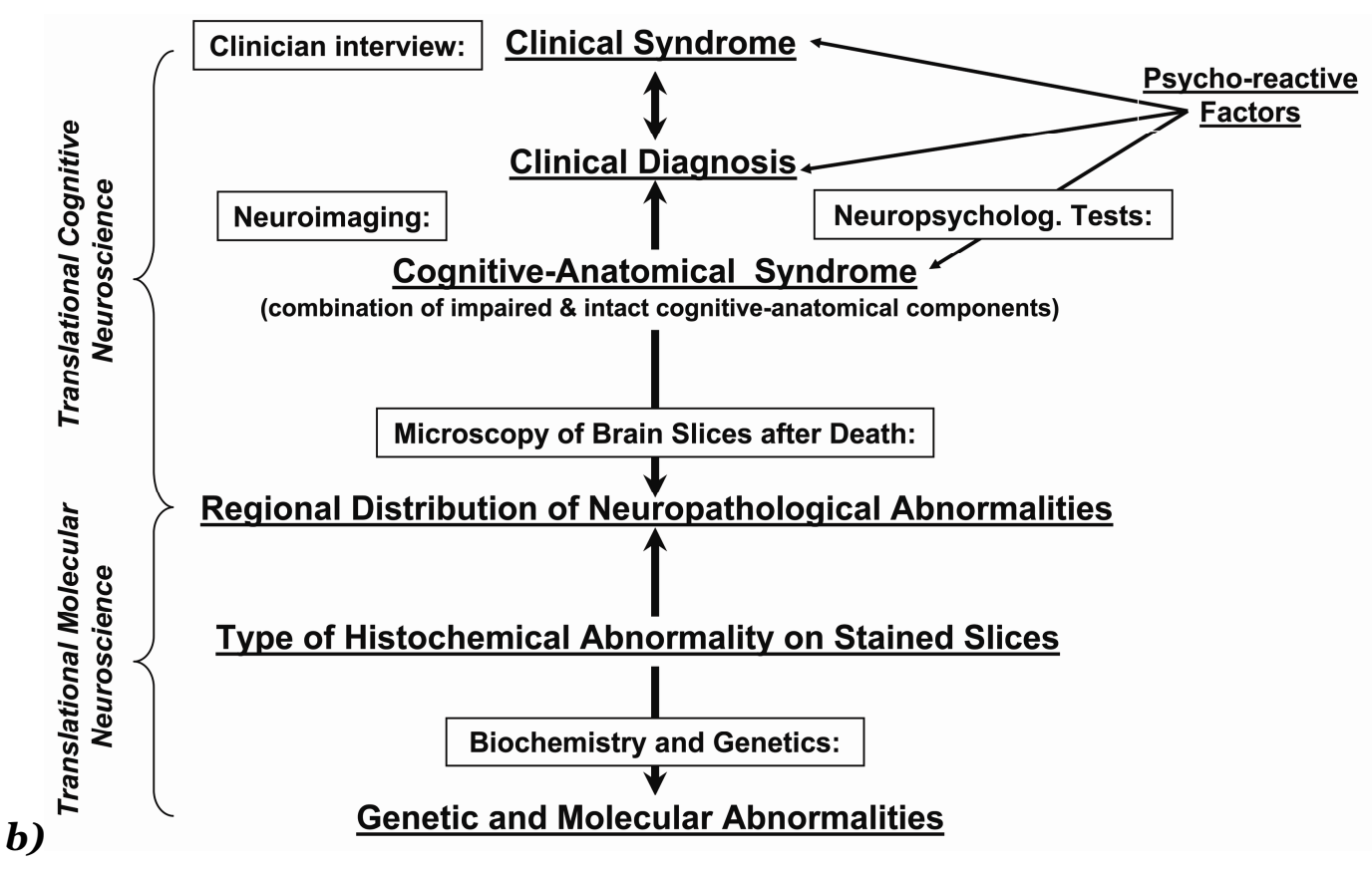

Fig. 2. Panels $a$ and $b$ illustrate the different approaches of dual-level translational neuroscience models (Fig.2a) and the proposed multi-level translational cognitive neuroscience models (Fig.2b). The relationship between clinical syndrome, clinical diagnosis and cognitive-anatomical syndrome is not only influenced by the underlying neurobiological causes, but also by the socio-cultural and diagnostic norms which define abnormal psychosocial functioning as well as the patient's and 
carer's experience of symptoms and degree of suffering. Cognitive-anatomical variation does not only capture neurobiologically induced changes (e.g. intrauterine infections, genetic or hormonal factors), but also changes induced by learning and psycho-reactive factors such as psycho-traumata which change the neurobiological and cognitive architecture through learning. Cognitive-anatomical variation is not restricted to macrostructural anatomical variation, but could also relate to differences in microstructural and connectivity variations. Extensions of the cognitive-anatomical approach can also include electrophysiological and neurochemical variations in particular brain networks.

\section{The role of neuroimaging in modelling the pathogenesis of inappropriate social behaviour in frontotemporal dementia syndromes}

In order to illustrate the translational cognitive neuroscience approach, its application to the understanding of the cognitive-anatomical basis of inappropriate social behaviour is described here. Slowly progressive inappropriate social behaviour, slowly starting in later adult life is an early, prominent and distinctive symptom of the behavioural variant of FTD $^{21}$. In contrast, inappropriate social behaviour does not occur in early forms of typical Alzheimer's dementia which is characterized by early prominence of impaired recent memory, which is intact in early behavioural variant FTD as is spatial orientation.

A translational cognitive neuroscience approach to inappropriate social behaviour in behavioural variant FTD is depicted in Figure 3. 


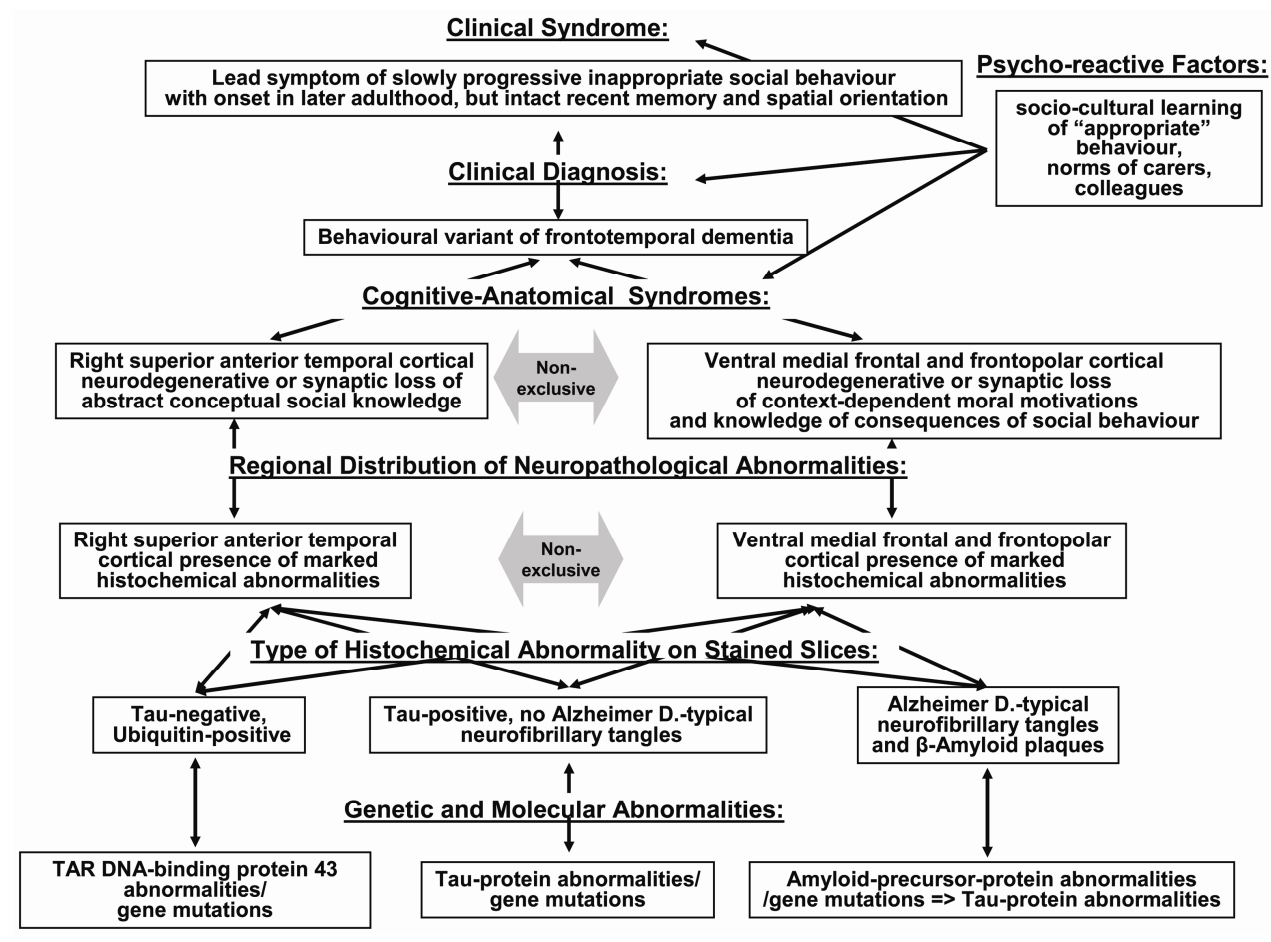

Fig. 3. A translational cognitive neuroscience approach to inappropriate social behaviour in the behavioural variant of FTD is illustrated. Examples for each level are given: clinical syndrome, clinical diagnosis, cognitive-anatomical syndrome, regional distribution of neuropathological abnormalities, type of histochemical abnormalities and genetic and molecular abnormalities. Standard neuroimaging (e.g. CT and MRI) plays an important role for establishing the clinical diagnosis by ruling out non-neurodegenerative causes of the disease. Advanced neuroimaging (using quantitative analysis of high-resolution 3-dimensional T1-weighted images (e.g. 25), diffusion tensor imaging (26), arterial spin labelling (e.g. ${ }^{27}$ ), perfusion MRI (e.g. 5), MR-spectroscopy (e.g. ${ }^{25}$ ), or 18-Fluorodeoxy-glucose PET (e.g. ${ }^{28}$ ) and neuropsychological test examination (here the Social conceptual discrimination task, ${ }^{29}$ together with standard tests) can be used to identify the pattern of intact and impaired cognitive components and of intact and abnormal metabolism and structure of grey-matter and white-matter. Please note that the depicted histochemical and molecular associations have not been directly tested with the described cognitive-anatomical syndromes and are only for illustrative purposes.

The clinical syndrome consists of the lead deficit symptom (i.e. an early, prominent and distinctive symptom of the syndrome) together with other important intact abilities and the time-course and onset of the symptom. The same symptom can occur in different clinical syndromes varying in associated symptoms, relative prominence, or time-course. For example, inappropriate social behaviour may occur as part of a manic syndrome in late-onset 
bipolar disorders, one of the most important differences when compared with FTD would be the phasic vs. slowly progressive time-course of the syndrome. Inappropriate social behaviour can also co-occur with prominent word finding and comprehension problems, but with the naming problems preceding the social behavioural impairment. This clinical syndrome would be characteristic for patients diagnosed with another form of FTD, namely semantic dementia $^{30}$. Another clinical presentation of FTD, the right hemispheric variant of semantic dementia was observed to show impairments of face and object recognition together with behavioural changes ${ }^{31}$. Here I focus on the syndrome of slowly progressive inappropriate social behaviour without prominent early impairments of either comprehension or object recognition occurring in later life.

The clinical syndrome itself is not a clinical diagnosis, because the syndrome makes no aetiological assumptions, although clinical syndromes are always influenced by clinical diagnostic classifications in practice. The clinical diagnosis according to the Lund-Manchester consensus criteria ${ }^{21}$ can only be made when a neurodegenerative cause of the syndrome can be assumed. The exact type of clinical history, additional laboratory tests and clinical neuroimaging required to rule out non-neurodegenerative causes, such as tumours for example, varies with time and clinical setting. But the step from a clinical syndrome to a clinical diagnosis is always one which requires additional aetiological considerations. Both clinical syndrome and clinical diagnosis are influenced by psycho-reactive factors (i.e. partially caused by a reaction to psychological experiences including the socio-cultural environment and emerging from an interaction with the socio-cultural environment). Appropriate social behaviour, for example, is culture-specific and the tolerance level and reaction of carers and work colleagues will largely affect how psychosocial functioning of a patient is perceived and when carers or work colleagues will decide that psychosocial functioning is abnormal.

The same clinical syndrome within a certain clinical diagnosis can nevertheless be due to different cognitive-anatomical syndromes as we have recently demonstrated for inappropriate social behaviour in patients with FTD ${ }^{29}$. We were able to show that inappropriate social behaviour was associated with loss of abstract conceptual knowledge of social behaviour (e.g. what it means to behave 'politely' or 'generously'). Further, this loss of abstract social knowledge was associated with hypometabolism (measured with 18-FDG-PET) within the right superior anterior temporal lobe. Many patients with selective impairments on abstract conceptual knowledge of social behaviour, but intact other conceptual knowledge (for example knowledge of animal behaviour and properties: e.g. 'nutritious', 'trainable') had 
the clinical diagnosis of behavioural variant FTD. This means that word finding problems and general comprehension had not been the lead symptom of their disease and thus they hadn't been classified as semantic dementia which is a clinical diagnosis associated with bilateral, especially inferior/middle anterior temporal lobe atrophy.

It is known, however, that ventral medial frontal lobe atrophy is associated with inappropriate social behaviour in patients with FTD as well ${ }^{32}$. The exact cognitive function of the ventral medial frontal lobes are currently debated, but there is evidence from fMRI in healthy human subjects that frontopolar sectors of the ventral medial frontal cortex may be representing complex (con-)sequences of behaviour33 and that subgenual sectors of the ventral medial frontal cortex (extending into the septal area 34 ) may be representing affiliative rewards and punishments enabling altruistic motivations 35,36 . The inability to foresee future consequences of one's behaviour or lack of altruistic concern may lead to inappropriate social behaviour as does lack of conceptual social knowledge and current behavioural interviews are unable to distinguish the resulting behaviour. Based on our cognitive-anatomical model of social and moral behaviour37, we would therefore propose three dissociable cognitiveanatomical components which can lead to similar behavioural symptoms and can combine with different weightings within different cognitive-anatomical syndromes (e.g. impairment of right anterior temporal cortical abstract conceptual social knowledge, impairment of (septal)-subgenual cortical affiliative reward/punishment values of social behaviour, impairment of frontopolar cortical representations of complex (con-)sequences of social behaviour) associated with the same clinical diagnosis of behavioural variant of FTD (please see Fig. 4 for cognitive-anatomical correlations). In most patients with the behavioural variant of FTD, the latter two components may be associated and therefore I have grouped them into one cognitive-anatomical syndrome. To further complicate the picture, there will be different degrees of overlap between the cognitive-anatomical syndromes, because patients with right superior anterior temporal lobe hypometabolism often have lesions to the ventral frontal cortex as well 29,38 . 


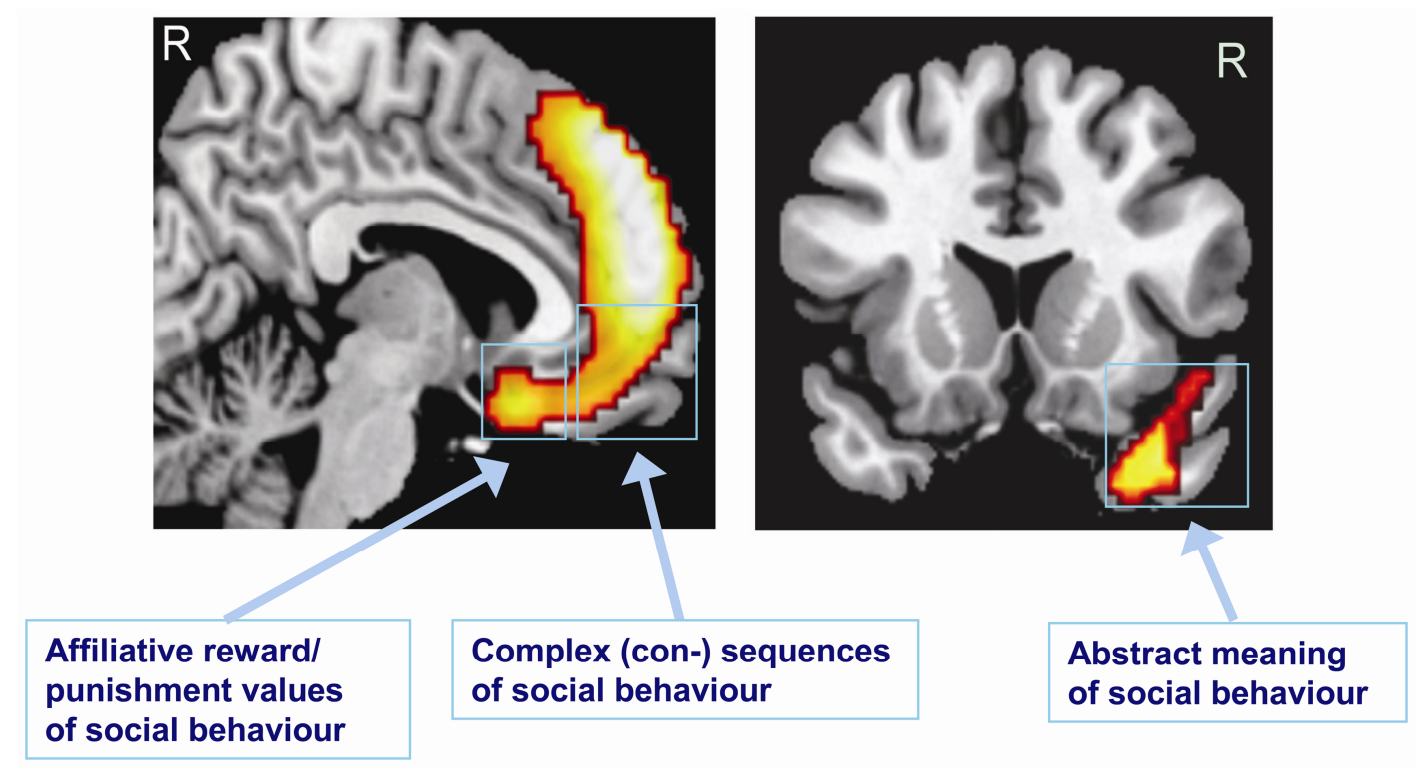

Fig. 4. The left panel shows consistent hypometablism in the medial prefrontal cortex (including the subgenual region, the ventral medial frontopolar and dorsal medial prefrontal cortex) in a group of patients with FTD compared with aged healthy controls and a group of patients with cortico-basal syndrome on FDG-PET. The right panel shows hypometabolism associated with selective impairments on social conceptual knowledge when compared with knowledge of less socially relevant concepts (reprinted with permission from 27). Hypothesized cognitive-anatomical components damaged by synaptic or neuronal dysfunction that contribute to inappropriate social behaviour are depicted with arrows: 1) Subgenual cortical representation of affiliative reward and punishment values of social behaviour/actions, necessary for altruistic concern30-32, 48, 2) Frontopolar cortical representation of complex (con-)sequences of social behaviour $\left.{ }^{29}, 33,56,3\right)$ Right anterior temporal representation of abstract conceptual meaning of social behaviour27, 33, 48, 63. Orbitofrontal and ventral parts of the cingulate cortex have been implicated in representations of reward and punishment values more generally ${ }^{64}$ and the bilateral anterior temporal lobes have been associated with amodal conceptual knowledge representations ${ }^{65}$ and conceptual differentiation and generalization ${ }^{66}$ for any type of concept. The cognitive-anatomical model proposed here is adapted from $^{32}, 33$ and assumes topographic distribution of representations according to the similarity of represented contents within the ventral cingulate, anterior temporal and frontopolar cortex. This topographic distribution is able to explain why subregions can be specialized for representing certain types of rewards/punishment associations, conceptual qualities, and action (con-) sequences.

On the next level of description, in vivo brain imaging results are correlated with postmortem inspection of the regional distribution of abnormalities including atrophy and histochemical changes on stained slices. In a post-mortem study, Snowden et al. ${ }^{31}$ for 
example showed that there was combined frontal and anterior temporal atrophy in 30 out of 39 cases with behavioural variant FTD, but the remaining 9 cases showed either predominant frontal or temporal changes. This demonstrates that the same clinical diagnosis can be associated with differences in regional distribution of neuropathological changes.

At a separate level of description, the histochemical type of abnormality can be further characterized. In Snowden et al's study, for example, there were about half of the behavioural variant FTD patients showing tau-positive abnormalities and the other half showing taunegative, ubiquitin-positive changes. None of the patients showed AD-typical neurofibrillary tangles and beta-amyloid plaques. I have included AD-typical histochemical abnormalities here, however, because Alladi and colleagues found 2 such cases in a behavioural variant FTD group of $28^{19}$. New developments in molecular imaging using either PET-ligands such as ligands for beta-amyloid 39 will allow direct in vivo assessment of histochemical and molecular abnormalities and will allow to study correlations between different regional distributions of pathology and the type of histochemical change.

At the lowest level of aetio-pathogenetic description, some of the genetic and molecular changes associated with certain histochemical abnormalities are illustrated. In the study by Snowden et al.31, for example, 10 out of 39 patients with the behavioural variant of FTD exhibited tau gene mutations and abnormalities in the tau protein. These tau abnormalities may also occur with normal genes due to changes of the translated protein and cause taupositive histochemical changes in a subset of FTD cases and in $\mathrm{AD}^{40}$. Ubiquitin-positive, taunegative cases have been linked with abnormalities in the TAR DNA-binding protein 43 and a subset of those cases show mutations in the progranulin gene ${ }^{18}$. The aim of translational molecular neuroscience according to this scheme is to identify the molecular pathogenesis and link molecular changes to the regional distribution of neuropathology, this can be achieved by molecular neuroimaging. Translational cognitive neuroscience would then aim to link regional distribution of neuropathological changes with cognitive-anatomical syndromes, clinical diagnoses and ultimately the clinical syndrome and symptoms. Non-molecular advanced quantitative neuroimaging techniques together with neuropsychological tests specifically allowing to dissociate cognitive components with anatomical specificity are necessary to achieve this translation. 


\section{The role of neuroimaging in modelling the pathogenesis of disorders without gross macro-anatomical abnormalities}

The previous section has described the use of structural and metabolic resting state imaging to identify cognitive-anatomical components underlying neuropsychiatric symptoms associated with marked and consistent macroanatomical abnormalities. Although functional activation imaging such as fMRI can be used in patients with brain lesions, it should be reserved for studying the neuroanatomy of retained or recovered functions rather than impaired abilities ${ }^{41}$. This is because imaging during a task which a patient cannot perform does not tell us whether abnormal activation is due to inability to do the task or dysfunction of particular brain regions ${ }^{42}$.

Many neuropsychiatric disorders, such as major depressive disorder (MDD), are not associated with consistent and marked macro-anatomical abnormalities such that on visual inspection structural MRIs appear within the expected normal variability. Functional MRI methods are therefore of particular interest for the study of such disorders. Resting state metabolism or perfusion can be informative, but findings are often inconsistent. One reason for inconsistencies may be the heterogeneity of diagnostic groups. For example, MDD patients with a family history of MDD may show more consistent abnormalities in the subgenual cingulate region than mixed groups of non-familial and familial MDD ${ }^{43}$. But there are core symptoms of MDD occurring in all subtypes and the first step in building cognitiveanatomical models of psychiatric disorders is to choose a suitable symptom-complex (i.e. clinical syndrome) within the broader range of symptoms for modelling. Such an approach has been recently taken to postulate a cognitive-anatomical model of obsessive-compulsive disorder 44 .

As Karl Jaspers, the founder of phenomenological psychopathology at the beginning of the $2 \mathrm{O}^{\text {th }}$ century, noted on the analyses of symptom-complexes $\left({ }^{9}, \mathrm{p} 582 \mathrm{ff}\right)$ there are different aspects of the relation of symptoms within a symptom-complex: 1) frequency of symptom cooccurrence, 2) coherence of symptoms by being related to a common aspect or function and 3) primary symptoms caused by the aetio-pathogenetic process and secondary symptoms emerging from these in an understandable way.

The second aspect, that of symptom coherence has been emphasized by Carl Schneider (as reviewed by Karl Jaspers) arguing about symptoms: "Their connectedness must be due to a normal complex of psychic function, which complex has been affected by the illness". At the 
time of this theory a lack of knowledge about neurobiologically valid models of many higher cognitive functions hampered the success of this approach. In my view, it is worth trying to go this way again, now that we have a more advanced knowledge of what cognitive functions behind symptoms to look for. In order to do that, however, we need to start by isolating symptom-complexes which are likely to be associated with a restricted set of cognitiveanatomical syndromes.

The core symptoms of major depression when assessed in a large World Health Organization (WHO) multicenter study using semi-structured interviews and international consensus criteria across different cultures and languages were: sadness, joylessness, anxiety and tension, lack of energy, loss of interests, experience of loss of the ability to concentrate, and ideas of insufficiency, inadequacy and worthlessness 45 . These core symptoms were present for endogenous (now operationalized as the "melancholic" subtype in DSM-IV) and nonendogenous forms of major depression, a distinction which is hotly debated ${ }^{46}$. Although sad/low mood is one of the most sensitive symptoms, it is questionable whether this is the primary symptom of MDD. This is because sad mood occurs in normal life and grief as well. Depressive sadness, however, has a distinct quality in patients with severe forms of acute MDD when compared with healthy sadness due to loss; furthermore sadness in depressive episodes is characteristically present independently of evoking situations. Persistent ideas/feelings of worthlessness, as distinct from low self-esteem, do not occur outside of depressive episodes and could explain persistently low mood. Therefore, the experience of worthlessness is a plausible candidate for a primary symptom of MDD.

One cognitive model of MDD, the learned helplessness model, explains vulnerability to major depressive episodes by internal, global and stable attributions for failure ${ }^{47}$. This means that people with a vulnerability to MDD tend to overgeneralize failure in one aspect or situation to their person as a whole across all future situations and aspects and take full responsibility for all failures instead of partly blaming them on external circumstances which can explain feelings of worthlessness and hopelessness. Cognitive therapy, proven to be effective in randomized controlled clinical trials, aims at changing the cognitive style of people with MDD which is characterized by automatic overgeneral negative attitudes towards oneself 48 .

Intuitive causal attributions of blame result in moral feelings such as guilt, shame, or selfcontempt (self-blame) and indignation/anger or contempt/disgust towards others (otherblame). Berrios has used a sensitive self-report scale to demonstrate that guilt and shame are significantly increased in people with different forms of major depression ${ }^{49}$. O'Connor et al. 
pointed to the link of increased interpersonal guilt and altruistic concern in major depression and developed a novel self-report questionnaire (Interpersonal Guilt Questionnaire ${ }^{50}$ ). Inappropriate guilt, when strictly defined, only occurs in a subset of patients with acute MDD45. Feelings of worthlessness, however, could be another expression of self-blame when applied to failure to achieve personal goals or live up to important values which do not necessarily cause interpersonal harm. This is true of some instances of shame or selfcontempt which do not require interpersonal harm or moral value violation as is usually the ingredient of guilt-evoking stimuli ${ }^{51,52}$.

Recent studies point to the selective importance of the subgenual cingulate cortex for selfblame when controlling for other-blame and unpleasantness ${ }^{52}$. Further, the frontopolar cortex has been shown to be more strongly activated for prosocial moral feelings (embarrassment, compassion, guilt) when compared with other-blaming feelings (indignation/anger towards others) ${ }^{51}$. In addition, there is evidence that individuals differ with respect to their degree of subgenual cingulate activation in response to guilt-evoking imagined moral violations $5^{2}$ and that individuals with higher altruistic concern show stronger activations while feeling guilty within this area35. Other-blaming feelings such as indignation/anger or contempt-disgust towards others were associated with dorsal anterior cingulate, lateral orbitofrontal, anterior insular, and dorsolateral prefrontal activations ${ }^{51,52}$. This potential functional subdivision within the frontal cortex for self- and other-blaming feelings could explain the dissociations between self- and other-blaming negative feelings observed in MDD.

Interestingly, the subgenual cingulate cortex is one area of reproducible abnormality in people with MDD. Resting state functional imaging (FDG-PET, SPECT) shows increased metabolism/perfusion in this area when correcting for partial volume effects due to reductions in grey matter persisting through asymptomatic and symptomatic phases of the illness ${ }^{43}$. Moreover, deep brain stimulation within the SCC was shown to be effective in therapy-resistant depression and leading to a normalization of FDG-PET metabolism ${ }^{53}$. Postmortem neuropathological studies revealed reductions in glial cells with intact neurons explaining the subgenual cingulate grey-matter reduction ${ }^{43}$.

Although it would be tempting to postulate a cognitive-anatomical model of MDD based on the above data in which one would explain increased tendencies for self-blame by dysfunction within the subgenual cingulate cortex, there is additional evidence which needs to be integrated. Firstly, grey matter reductions within the subgenual cingulate cortex are not 
specific for MDD, but can be found in patients with bipolar disorder as well43, this means that the abnormalities within this region could explain vulnerability for inappropriate self-blame in the depressive phases occurring in both disorders, but the emergence of depressive phases can clearly not solely be explained on the basis of abnormality in a single brain region. Secondly, abnormalities in several other frontal, anterior temporal and subcortical regions emerge from a meta-analysis of resting state FDG-PET, SPECT or fMRI using positive and negative emotional stimuli in MDD54.

One promising approach to understand the multitude of brain regions showing subtle metabolic/perfusion abnormalities in MDD has been the model of disrupted interaction between brain regions in a fronto-limbic network55. Using structural equation modelling, Seminowicz et al. were able to confirm the importance of effective connectivity within a network of regions of interest on FDG-PET. This network included, the right hippocampus, right anterior thalamus, medial orbitofrontal (BA11), ventral medial frontopolar cortex (BA10), left dorsolateral prefrontal cortex (BA9), pregenual anterior cingulate cortex (BA24) and posterior subgenual cingulate cortex (BA25). Disruption of functional integration within a network of areas connected with the subgenual cingulate cortex has also been demonstrated using resting state BOLD imaging 56 .

A suitable cognitive-anatomical model of MDD will therefore need to account for the involvement of above network of brain regions (and potentially others as well) in phases of MDD and how vulnerability for MDD leads to phases of depression disrupting this network. The model needs to explain how inappropriate overgeneralizations of self-blame, but not blame of others, can occur in the depressive phase. The model further needs to explain which part of the brain network is linked to this symptom and whether overgeneralized self-blame can indeed serve as a primary cause of other symptoms of depresssion, or whether for example somatic symptoms such as loss of vitality, early awakening and appetite loss need to be explained by a separate mechanism. Further, the influence of learning, such as through early childhood experiences, and the influence of neurochemical mechanisms such as the known abnormalities within for example the serotonergic system ${ }^{57}$ need to be integrated in order to explain disruption of functional integration within the network.

In contrast with translational cognitive neuroscience models of dementia, the molecular and genetic level of description may not be easily related with post-mortem neuropathological changes. Instead, molecular neuroimaging using PET-ligands ${ }^{17}$, pharmaco-MRI ${ }^{8}$ and genetic 
investigations ${ }^{14}$ are more promising as tools to link the cognitive-anatomical syndrome level with molecular pathogenetic factors.

Despite the exciting advances in neurochemical and genetic methods, translational applications of these techniques cannot avoid the challenge of formulating and testing comprehensive cognitive-anatomical models that are able to account for primary symptoms of MDD. Current cognitive-anatomical models of increased negative emotions due to lack of prefrontal suppression of limbic-mediated emotion ${ }^{59}$ cannot account for the clinical observation that people with acute MDD feel badly about themselves, but do not typically show an overall increase in negative feelings towards others (i.e. anger towards others).

\section{Summary and future perspectives}

Here, a multilevel approach to translational neuroscience, referred to as translational cognitive neuroscience has been described which stresses the importance of cognitiveanatomical syndromes as an intermediate level of aetio-pathogenetic inference in the understanding of neuropsychiatric disorders. This approach builds on previous frameworks on how to integrate cognitive neuroscience methods into clinical and translational neuropsychiatry3, 14. The introduction of two main claims which are in contrast with previous approaches aimed at stimulating a nosologically driven debate about suitable frameworks for translational neuroscience.

The first claim is that rather than seeking a purely cognitive description of impaired and intact functions, we should use advanced neuroimaging to derive cognitive components which are associated with functionally specialized brain regions and successively replace cognitive models with elusive anatomical specificity. The prediction is that these functionally specialized brain regions will not be specific to any given task, but integration of information across networks of regions with different specializations is required for any task which can account for partial redundancy for a given task within the network ${ }^{41}$. Therefore, in order to decompose functional specializations within a network the suggestion is to go beyond describing the input and output of a brain region and to identify the type of representation in a particular area (for a review of representational versus processing models of e.g. the prefrontal cortex see ${ }^{60}$ ). 
The second main claim is that translational neuroscience should not aim at reducing the number of levels of aetio-pathogenetic inference, and should allow for one-to-many or manyto-one relationships between categories within adjacent levels. By nature of the aetiopathogenetic process, it will be more likely to find high associations between one category at one level with another category at an adjacent level (molecular and histopathological) rather than finding associations between lowest and highest levels of description (e.g. molecular \& symptom). According to the proposed approach here, this problem cannot, however, be solved by eliminating the syndromic and diagnostic levels of description and by only focussing on neuroanatomical and molecular categorization, because clinical symptoms or syndromes will not map onto neurobiological changes in a predictable one-to-one way. I have further argued that the phenomenological symptom-level of understanding neuropsychiatric disorders is irreplaceable, because only symptoms and the partly subjective or culturallyshaped thresholds entailed in symptoms can indicate the pathological value (i.e. degree of suffering caused) of a neuroanatomical or cognitive abnormality.

In order to fully understand the relationship between symptoms and cognitive or anatomical abnormalities, one needs to study a control population which has not been selected on the basis of the presence of symptoms, but was selected on the basis of the cognitive or anatomical abnormality instead, a methodological limitation of most clinical studies. Neuroanatomical and cognitive abnormalities can occur in populations free of symptoms and may point to important individual variations in the cognitive-anatomical architecture of a given system or to important personal psycho-reactive or socio-cultural variations in the learning history of people which are likely to have a large influence on the cognitiveanatomical architecture. Teuber ${ }^{61}$ reviews one of the largest studies avoiding selection bias by symptoms and using only lesion criteria for patient selection, carried out by Feuchtwanger in 1923, who conducted a systematic prospective assessment of 200 frontal and 200 non-frontal gunshot wounds during world-war I. Interestingly, he found 13/200 frontal lesion cases with no symptoms at all. Modern neuroimaging would have been able to give us a more accurate measure of the size and location of lesions in these 13 patients, but even when using in vivo neuroimaging, the variability of symptoms in response to the same type and size of lesion is remarkable and puzzling62. These observations of variability in the associations of symptoms, cognitive impairments and neuroantomical changes point to the irreducibility of multi-level approaches in translational neuroscience.

An exciting future of translational cognitive and molecular neuroscience research lies ahead of us, in which we will be able to combine detailed phenomenological assessments of 
symptoms with experimental neuropsychological paradigms, high-resolution multimodal neuroimaging, and molecular and genetic methods to unravel the complex mechanisms which cause neuropsychiatric disorders. This will allow translational neuroscience to find new mechanisms for pharmacological, cognitive and behavioural treatment and new ways of early diagnosis and prevention to improve the lives of patients and carers.

\section{Acknowledgements}

I thank Jorge Moll for helpful comments on the manuscript. The author was supported by a Stepping Stones Fellowship from the Faculty of Medical and Human Sciences \& Insitute of Health Sciences, The University of Manchester, Manchester, UK. 


\section{References}

1. Science Translational Medicine.

http://www.sciencemag.org/marketing/stm/definition.dtl. Accessed 5/23/09, 2009.

2. Kendler KS. Explanatory models for psychiatric illness. American Journal of Psychiatry. Jun 2008;165(6):695-702.

3. Halligan PW, David AS. Cognitive neuropsychiatry: towards a scientific psychopathology. Nature Reviews Neuroscience. Mar 2001;2(3):209-215.

4. Shallice T. From Neuropsychology to Mental Structure. Cambridge: Cambridge University Press; 1990.

5. DeLeon J, Gottesman RF, Kleinman JT, et al. Neural regions essential for distinct cognitive processes underlying picture naming. Brain. May 2007;130:1408-1422.

6. Gazzaniga MS. The Cognitive Neurosciences. 3 ed. Boston: MIT Press; 2004.

7. WHO. International Classification of Disorders, Chapter V, Mental and behavioural disorders. http://apps.who.int/classifications/apps/icd/icd10online/.

8. Widiger TA, Samuel DB. Diagnostic categories or dimensions? A question for the diagnostic and statistical manual of mental disorders-fifth edition. Journal of Abnormal Psychology. Nov 2005;114(4):494-504.

9. Jaspers K. General Psychopathology. 7 ed. Chicago: Chicago University Press; $1963 / 1959$.

10. Busch H, Cranach MV, Gulbinat W, Renfordt E, Tegeler J. Reliability of the AmdpSystem - a Preliminary-Report on a Multicenter Exercise on the Reliability of Psychopathological Assessment. Acta Psychiatrica Scandinavica. 1980;62(4):382-391.

11. First MB, Gibbon M, Spitzer RL, Williams JBW. S C I D - I, Structured Clinical Interview for DSM-IV-TR axis I Disorders

New York: Biometrics Research; 2002.

12. Cummings JL, Mega M, Gray K, Rosenbergthompson S, Carusi DA, Gornbein J. The Neuropsychiatric Inventory - Comprehensive Assessment of Psychopathology in Dementia. Neurology. Dec 1994;44(12):2308-2314.

13. Kendler KS. Reflections on the relationship between psychiatric genetics and psychiatric nosology. American Journal of Psychiatry. Jul 2006;163(7):1138-1146. 
14. Meyer-Lindenberg A, Weinberger DR. Intermediate phenotypes and genetic mechanisms of psychiatric disorders. Nature Reviews Neuroscience. Oct 2006;7(10):818-827.

15. Small GW. Diagnostic issues in dementia: Neuroimaging as a surrogate marker of disease. Journal of Geriatric Psychiatry and Neurology. Sep 2006;19(3):180-185.

16. Matthews PM, Honey GD, Bullmore ET. Applications of fMRI in translational medicine and clinical practice. Nature Reviews Neuroscience. Sep 2006;7(9):732-744.

17. Wong DF, Tauscher J, Grunder G. The Role of Imaging in Proof of Concept for CNS Drug Discovery and Development. Neuropsychopharmacology. Jan 2009;34(1):187203.

18. Josephs KA. Frontotemporal dementia and related disorders: Deciphering the enigma. Annals of Neurology. Jul 2008;64(1):4-14.

19. Alladi S, Xuereb J, Bak T, et al. Focal cortical presentations of Alzheimer's disease. Brain. Oct 2007;130:2636-2645.

20. Zahn R, Burns A. Dementia disorders: an overview. In: Waldemar G, Burns A, eds. Alzheimer's Disease. Oxford: Oxford University Press; 2009.

21. Neary D, Snowden JS, Gustafson L, et al. Frontotemporal lobar degeneration: a consensus on clinical diagnostic criteria. Neurology. Dec 1998;51(6):1546-1554.

22. Waldemar G, Dubois B, Emre M, et al. Recommendations for the diagnosis and management of Alzheimer's disease and other disorders associated with dementia: EFNS guideline. Eur. J. Neu. 2007;14:e1-e26.

23. Ralph MAL, Lowe C, Rogers TT. Neural basis of category-specific semantic deficits for living things: evidence from semantic dementia, HSVE and a neural network model. Brain. Apr 2007;130:1127-1137.

24. Pereira JMS, Williams GB, Acosta-Cabronero J, et al. Atrophy patterns in histologic vs clinical groupings of frontotemporal lobar degeneration. Neurology. May 12 2009;72(19):1653-1660.

25. Zahn R, Buechert M, Overmans J, et al. Mapping of temporal and parietal cortex in progressive nonfluent aphasia and Alzheimer's disease using chemical shift imaging, voxel-based morphometry and positron emission tomography. Psychiatry Research: Neuroimaging. 2005/11/30 2005;140(2):115-131.

26. Bozzali M, Cherubini A. Diffusion tensor MRI to investigate dementias: a brief review. Magnetic Resonance Imaging. Jul 2007;25(6):969-977. 
27. Du AT, Jahng GH, Hayasaka S, et al. Hypoperfusion in frontotemporal dementia and Alzheimer disease by arterial spin labeling MRI. Neurology. Oct 10 2006;67(7):12151220.

28. Salmon E, Kerrouche N, Herholz K, et al. Decomposition of metabolic brain clusters in the frontal variant of frontotemporal dementia. Neuroimage. 2006;30(3):871-878.

29. Zahn R, Moll J, Iyengar V, et al. Social conceptual impairments in frontotemporal lobar degeneration with right anterior temporal hypometabolism. Brain. January 19, 2009 2009;132(3):604-616.

30. Bozeat S, Gregory CA, Ralph MAL, Hodges JR. Which neuropsychiatric and behavioural features distinguish frontal and temporal variants of frontotemporal dementia from Alzheimer's disease? Journal of Neurology Neurosurgery and Psychiatry. Aug 2000;69(2):178-186.

31. Snowden J, Neary D, Mann D. Frontotemporal lobar degeneration: clinical and pathological relationships. Acta Neuropathologica. Jul 2007;114(1):31-38.

32. Liu W, Miller BL, Kramer JH, et al. Behavioral disorders in the frontal and temporal variants of frontotemporal dementia. Neurology. Mar 9 2004;62(5):742-748.

33. Krueger F, Moll J, Zahn R, Heinecke A, Grafman J. Event frequency modulates the processing of daily life activities in human medial prefrontal cortex. Cerebral Cortex. Oct 2007;17(10):2346-2353.

34. Moll J, Krueger F, Zahn R, Pardini M, de Oliveira-Souza R, Grafman J. Human fronto-mesolimbic networks guide decisions about charitable donation. Proc Natl Acad Sci U S A. Oct 17 2006;103(42):15623-15628.

35. Zahn R, de Oliveira-Souza R, Bramati I, Garrido G, Moll J. Subgenual cingulate activity reflects individual differences in empathic concern. Neuroscience Letters. 2009;457(2):107-110.

36. Moll J, De Oliveira-Souza R, Zahn R. The Neural Basis of Moral Cognition: Sentiments, Concepts, and Values. Ann NY Acad Sci. March 1, 2008 2008;1124(1):161-180.

37. Moll J, Zahn R, de Oliveira-Souza R, Krueger F, Grafman J. Opinion: the neural basis of human moral cognition. Nat Rev Neurosci. Oct 2005;6(10):799-809.

38. Snowden JS, Bathgate D, Varma A, Blackshaw A, Gibbons ZC, Neary D. Distinct behavioural profiles in frontotemporal dementia and semantic dementia. Journal of Neurology Neurosurgery and Psychiatry. Mar 2001;70(3):323-332.

39. Herholz K, Carter SF, Jones M. Positron emission tomography imaging in dementia. Br J Radiol. December 1, 2007 2007;80(Special_Issue_2):S160-167. 
40. Hernandez F, Avila J. Tauopathies. Cellular and Molecular Life Sciences. Sep 2007;64(17):2219-2233.

41. Zahn R, Schwarz M, Huber W. Functional activation studies of word processing in the recovery from aphasia. J Physiol Paris. Jun 2006;99(4-6):370-385.

42. Price CJ, Mummery CJ, Moore CJ, Frakowiak RS, Friston KJ. Delineating necessary and sufficient neural systems with functional imaging studies of neuropsychological patients. J Cogn Neurosci. Jul 1999;11(4):371-382.

43. Drevets WC, Savitz J. The Subgenual Anterior Cingulate Cortex in Mood Disorders. Cns Spectrums. Aug 2008;13(8):663-681.

44. Huey ED, Zahn R, Krueger F, et al. A Psychological and Neuroanatomical Model of Obsessive-Compulsive Disorder. Journal of Neuropsychiatry and Clinical Neurosciences. Fal 2008;20(4):390-408.

45. Sartorius N, Jablensky A, Gulbinat W, Ernberg G. WHO collaborative study: assessment of depressive disorders. Psychol Med. Nov 1980;10(4):743-749.

46. Parker G. Beyond major depression. Psychological Medicine. Apr 2005;35(4):467474.

47. Abramson LY, Seligman ME, Teasdale JD. Learned helplessness in humans: critique and reformulation. J Abnorm Psychol. Feb 1978;87(1):49-74.

48. Beck AT, Rush AJ, Shaw BF, Emery G. Cognitive Therapy of Depression New York: Guilford Press; 1979.

49. Berrios GE, Bulbena A, Bakshi N, et al. Feelings of Guilt in Major Depression Conceptual and Psychometric Aspects. British Journal of Psychiatry. Jun 1992;160:781-787.

50. OConnor LE, Berry JW, Weiss J, Bush M, Sampson H. Interpersonal guilt: The development of a new measure. Journal of Clinical Psychology. Jan 1997;53(1):73-89.

51. Moll J, de Oliveira-Souza R, Garrido GJ, et al. The self as a moral agent: linking the neural bases of social agency and moral sensitivity. Social Neuroscience. 2007;2(3 \& 4): $336-352$.

52. Zahn R, Moll J, Paiva M, et al. The Neural Basis of Human Social Values: Evidence from Functional MRI. Cereb. Cortex. February 1, 2009 2009;19(2):276-283.

53. Mayberg HS, Lozano AM, Voon V, et al. Deep brain stimulation for treatmentresistant depression. Neuron. Mar 3 2005;45(5):651-660.

54. Fitzgerald PB, Laird AR, Maller J, Daskalakis ZJ. A meta-analytic study of changes in brain activation in depression. Human Brain Mapping. Jun 2008;29(6):683-695. 
55. Seminowicz DA, Mayberg HS, McIntosh AR, et al. Limbic-frontal circuitry in major depression: a path modeling metanalysis. Neuroimage. May 2004;22(1):409-418.

56. Greicius MD, Flores BH, Menon V, et al. Resting-state functional connectivity in major depression: Abnormally increased contributions from subgenual cingulate cortex and thalamus. Biological Psychiatry. Sep 1 2007;62(5):429-437.

57. Deakin JFW. Depression and antisocial personality disorder: two contrasting disorders of 5HT function. Journal of Neural Transmission-Supplement. 2003(64):79-93.

58. McKie S, Del-Ben C, Elliott R, et al. Neuronal effects of acute citalopram detected by pharmacoMRI. Psychopharmacology (Berl). Aug 2005;180(4):680-686.

59. Ochsner KN, Bunge SA, Gross JJ, Gabrieli JDE. Rethinking feelings: An fMRI study of the cognitive regulation of emotion. Journal of Cognitive Neuroscience. Nov 15 2002;14(8):1215-1229.

60. Wood JN, Grafman J. Human prefrontal cortex: Processing and representational perspectives. Nature Reviews Neuroscience. Feb 2003;4(2):139-147.

61. Teuber HL. The Riddle of Frontal Lobe Function in Man (Reprinted from The frontal granular cortex and behavior, pg 410, 1964). Neuropsychology Review. Mar 2009;19(1):25-46.

62. Willmes K, Poeck K. To What Extent Can Aphasic Syndromes Be Localized. Brain. Dec 1993;116:1527-1540.

63. Zahn R, Moll J, Krueger F, Huey ED, Garrido G, Grafman J. Social concepts are represented in the superior anterior temporal cortex. Proc Natl Acad Sci U S A. Apr 10 2007;104(15):6430-6435.

64. Rolls ET, Grabenhorst F. The orbitofrontal cortex and beyond: From affect to decision-making. Progress in Neurobiology. Nov 2008;86(3):216-244.

65. Patterson K, Nestor PJ, Rogers TT. Where do you know what you know? The representation of semantic knowledge in the human brain. Nature Reviews Neuroscience. Dec 2007;8(12):976-987.

66. Ralph MAL, Patterson K. Generalization and differentiation in semantic memory Insights from semantic dementia. Year in Cognitive Neuroscience 2008.

2008;1124:61-76. 